\title{
Screening of up- and downregulation of circRNAs in HBV-related hepatocellular carcinoma by microarray
}

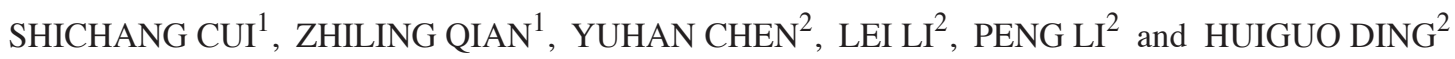 \\ ${ }^{1}$ Interventional Center for Oncology; ${ }^{2}$ Department of Hepatology and Gastroenterology, \\ Beijing You An Hospital, Capital Medical University, Beijing 100069, P.R. China
}

Received November 26, 2015; Accepted May 4, 2017

DOI: $10.3892 / 01.2017 .7265$

\begin{abstract}
The present study describes circular RNA (circRNA) profiles in three pairs of hepatocellular carcinoma (HCC) tissues and the corresponding adjacent non-tumorous tissues (NTs) by microarray. circRNA is a type of endogenous RNA that serve a crucial role in disease development and aberrantly express in a number of types of cancer. In the present study, 3 paired HCC tissues and paired adjacent NTs were collected from HCC surgical specimens from 3 hepatitis B virus-infected patients with HCC. With abundant and varied probes accounting for 5,396 circRNAs, a large number of circRNAs are able to be quantitatively determined. Based on the microarray data, 222,567,556 upregulated circRNAs and 125,439,219 downregulated circRNAs were identified respectively. Further analysis revealed 24 upregulated and 23 downregulated significantly circRNAs (fold-change $\geq 2$; $\mathrm{P} \leq 0.05)$ in HCC tissues compared with NTs. By means of computer analysis and database inquiring, the microRNA (miRNA) response elements associated with the abnormally expressed circRNAs were annotated. The present study showed novel evidence determining genome-wide circRNA expression patterns in HCC using microarray analysis. The results demonstrated that clusters of circRNAs were aberrantly expressed in HCC compared with NTs. These circRNAs may be involved in the occurrence and development of HCC. Therefore, the results of the present study may provide a novel approach for improving the understanding of the molecular basis of HCC. Furthermore, the identified circRNAs may be potential biomarkers for the diagnosis of HCC.
\end{abstract}

Correspondence to: Dr Huiguo Ding, Department of Hepatology and Gastroenterology, Beijing You An Hospital, Capital Medical University, 8 YouAnMenWai Street, Fengtai, Beijing 100069, P.R. China

E-mail: dinghuiguo@ccmu.edu.cn

Key words: hepatocellular carcinoma, circular RNA, microarray, hepatitis B virus, expression, biomarker

\section{Introduction}

Hepatocellular carcinoma (HCC) is the sixth most common type of cancer worldwide, ranking fifth and eighth in males and females, respectively, and exhibits one of the highest mortality rates (1). Hepatitis B virus (HBV) and hepatitis C virus (HCV) are primary causes of HCC. Chronic HBV infection is a dominant risk factor in the majority of areas of Asia and Sub-Saharan Africa that have a high incidence of HCC (2). The majority of patients with HCC whoexperience HBV infectionexhibit cirrhosis, secondary to the chronic necroinflammation (3). HBV, an oncogenic virus, promotes HCC via indirect (necroinflammation and regeneration injury) and direct (integration of its DNA in the host genome) pathways (4). The aberrant expression of genes and regulatory RNA moleculesare key nodes for the occurrence and development of HCC.

Circular RNAs (circRNA/ciR), initially observed in RNA viruses in the 1970s, have been identified as unique non-coding RNA molecules (5). CircRNAsare a type of endogenous RNA with a stable structure and tissue-specific expression (6) and are widely present in the cytoplasm of eukaryotic organisms, in the circular form (7). CircRNA forms a covalently closed continuous loop by means of unique non-canonical 'head-to-tail' splice without a free 3' or 5' end (8-10). CircRNAs derive from non-linear reverse splicing or gene rearrangement and circRNAs dominate the total spliced transcripts (11). High-throughput sequencing has enabled $>25,000$ types of circRNAs to be discovered in human fibroblasts (12). In addition, circRNAs may be formed in exons and introns, and cirRNAs with either origin may function in the regulation of gene expression (13).

A previous study demonstrated that circRNA served a role in the level of miRNA-mediated regulation of gene expression by sequestering the miRNAs. Furthermore, circRNAs are able to regulate gene expression by acting as competing endogenous RNAs and also termed miRNA 'sponges' (14). CircRNAs contain multiple, tandem miRNA binding sites. CircRNAs adsorb and sequester miRNAs to terminate the suppression of their targets, and to modulate the expression levels of other associated RNA molecules which share the same miRNA response elements (MREs) (14-16). The interaction between circRNAs and disease-associated miRNAs indicates that circRNAs are important for disease regulation (17). 
CircRNAs serve crucial roles in the development of diseases, including nervous system disorders and atherosclerosis $(18,19)$. In addition, circRNAs have been demonstrated to be involved in the neoplastic process (20); however, the molecular mechanisms underlying the association of circRNAs with cancer remain unclear (21).

To the best of our knowledge, a large-scale microarray screening of HCC and the focus of circRNAs as biomarkers of HCC has not been previously reported. The present study screened dysregulated circRNAs expression in HCC tissues using a microarray and annotated them for circRNA/microRNA (miRNA/miR) interactions.

\section{Patients and methods}

Patients and clinical specimens. The total three paired HCC tissues and adjacent non-tumorous tissues (NTs; Table I) were collected from HCC surgical specimens between June 2012 and December 2013 at Beijing YouAn Hospital, Capital Medical University (Beijing, China). All tissue specimens were immediately preserved in RNA-fixer reagent (BioTeke Corporation, Beijing, China) following removal from the body and were stored at $-80^{\circ} \mathrm{C}$ until use. The corresponding adjacent NTs were taken $5 \mathrm{~cm}$ from the edge of the cancer and contained no obvious tumor cells, as evaluated by an experienced pathologist.

All three HCC patients were diagnosed with HBV infection. Tumors were staged according to the tumor-node-metastasis (TNM) staging system (22). The three patients were diagnosed with T1N0M0, T1N0M0, and T3aN0M0, respectively (Table I). No radiotherapy, chemotherapy or targeted therapy was administered prior to surgery.

The present study protocol conforms to the ethical guidelines of the 1975 Declaration of Helsinki and was approved by the Ethics Committee of Beijing YouAn Hospital, Capital Medical University (Beijing, China). Written informed consent was obtained from all participants.

Total RNA extraction, labeling, hybridization, and array scanning. Total tissue RNA was extracted from the HCC tissues and paired adjacent NTs using TRIzol reagent (Invitrogen; Thermo Fisher Scientific, Inc., Waltham, MA, USA), following the manufacturer's protocol. CircRNAs were treated with RNase R (Epicentre; Illumina, Inc., San Diego, CA, USA) to remove linear RNAs, according to the manufacturer's protocol. Each sample was amplified and transcribed into fluorescent complementary RNA utilizing a random priming method (Arraystar Super RNA Labeling kit; Arraystar, Inc., Rockville, MD, USA). The labeled circRNAs were hybridized onto the Arraystar Human circRNA Array (5,396 human circRNA probes; cat. no. 6x7K; Arraystar, Inc.).

The labeled circRNAs were purified using an RNeasy Mini kit (Qiagen, Inc., Valencia, CA, USA). The concentration and specific activity of the labeled circRNAs (pmol Cy3/ $\mu$ g circRNA) were measured using NanoDrop ND-1000 spectrophotometer (NanoDrop; Thermo Fisher Scientific, Inc., Wilmington, DE, USA). A total of $1 \mu \mathrm{g}$ of each labeled circRNA was dispensed into the gasket slide and assembled to the circRNA expression microarray slide. The slides were incubated for $17 \mathrm{~h}$ at $65^{\circ} \mathrm{C}$ in an Agilent Hybridization Oven
(Agilent Technologies, Inc., Santa Clara, CA, USA). The hybridized arrays were washed, fixed and scanned using the Axon GenePix 4000B microarray scanner (Molecular Devices, Sunnyvale, CA, USA).

Detection of expression profiling data and differentially expressed data. Scanned images were imported into GenePix Pro version 6.0 software (Axon; Molecular Devices) for grid alignment and raw data extraction. Quantile normalization of raw data and subsequent data processing were performed using the $\mathrm{R}$ software package (version 3.1.2; Lucent Technologies, Inc.; Nokia, Espoo, Finland). Low-intensity filtering was performed, and the circRNAs with $\geq 2$ of the 6 samples having 'flags expressed' ( $\geq 2$ times background standard deviation) were retained for further analysis. The analysis outputs were filtered and the differentially expressed circRNAs were ranked according to fold-change and P-value. Differentially expressed circRNAs were filtered and illustrated as a volcano plot. Hierarchical clustering was performed to reveal the distinguishable circRNAs expression pattern among samples.

Annotation for circRNA/miRNA interaction. The circRNA/miRNA interaction was predicted using Target Scan (www.targetscan.org/vert_71) and Miranda (www.microrna. org/microrna/home.do). All differentially expressed circRNAs were annotated in detail using the circRNA/miRNA interaction information.

Statistical analysis. All data were analyzed using SPSS (version 21.0; IBM Corp., Armonk, NY, USA) and all results were presented as the mean \pm standard deviation. Differences between two groups were estimated using the Student's t-test, and fold-change $\geq 2.0$ and $\mathrm{P} \leq 0.05$ were considered to indicate a statistically significant difference.

\section{Results}

circRNA expression profiles. A total of 5,396circRNAs were scanned and the array image of each sample was demonstrated. Quantile normalization of raw data and subsequent data processing were performed using the $\mathrm{R}$ software package. The data demonstrated that 222,567,556 circRNAs were upregulated (fold-change $\geq 2$ ) and 125,439,219 circRNAs were downregulated (fold-change $\geq 2$; Fig. 1).

Differentially expressed circRNAs. The differentially expressed circRNAs with statistical significance between the two groups (HCC tissues group vs. NT group) were identified through using volcano plot filtering. A total of 24 upregulatedcircRNAs and 23 downregulated circRNAs were identified to be significant in HCC tissues compared with NTs (fold-change $\geq 2$; P $\leq 0.05$; Fig. 2; Tables II and III). The top five upregulated circRNAs were hsa_circRNA_104351, hsa_circRNA_102814, hsa_circRNA_103489, hsa_circRNA_102109 and hsa_circRNA_100381. Furthermore, the top five downregulated circRNAs were hsa_circRNA_100327, hsa_circRNA_101764, hsa_circR NA_101092, hsa_circRNA_001225 and hsa_ circRNA_102904. 
Table I. Clinical parameters of the three patients with hepatitis B-associated HCC.

\begin{tabular}{lcccccc}
\hline Patient & Sex & Age, years & HBV-DNA, IU/ml & Cirrhosis & Differentiation & HCC stage, TNM \\
\hline 1 & M & 63 & $4.01 \times 10^{6}$ & Yes & Middle & T1N0M0 \\
2 & M & 44 & $9.85 \times 10^{2}$ & Yes & Poor & T3aN0M0 \\
3 & F & 45 & $1.98 \times 10^{3}$ & Yes & Middle & T1N0M0
\end{tabular}

HCC, hepatocellular carcinoma; HBV, hepatitis B virus; TNM, tumor-node-metastasis; M, male; F, female.
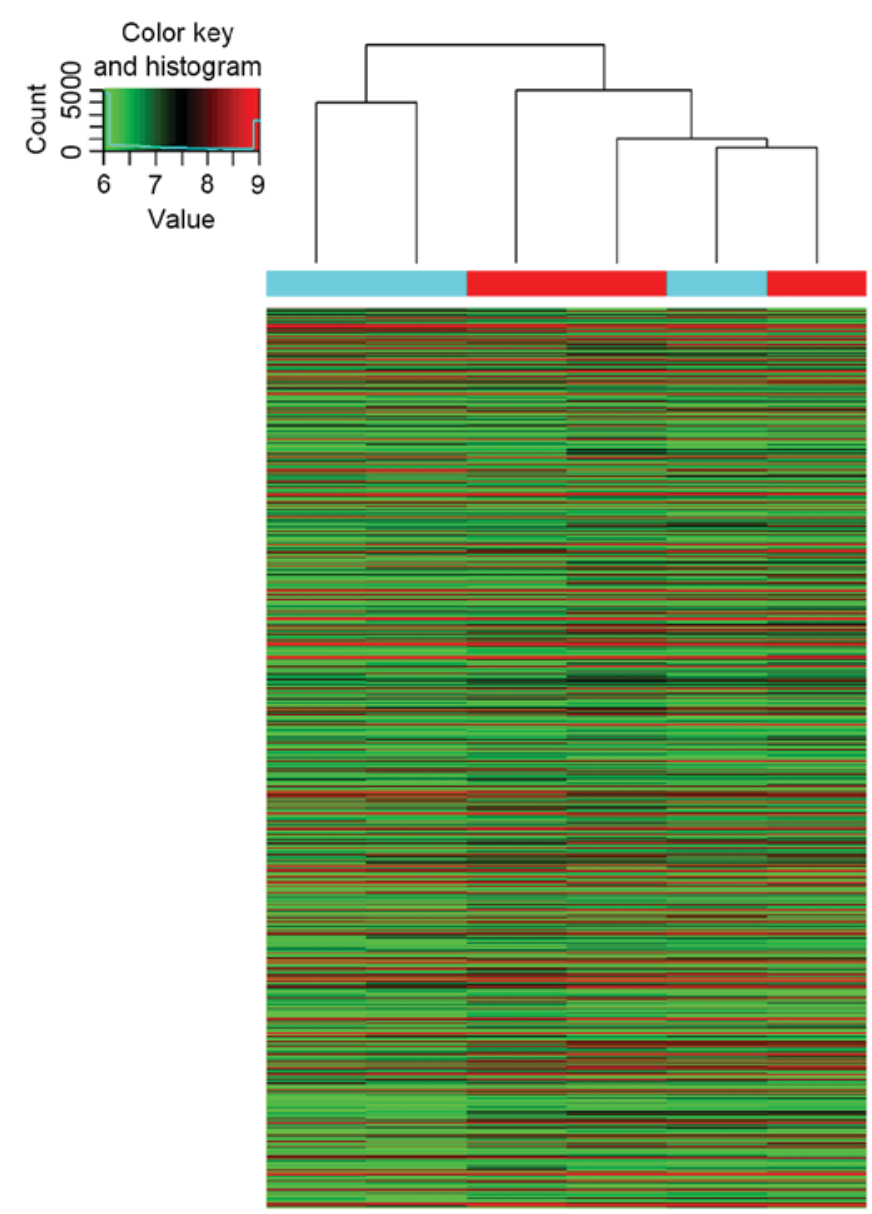

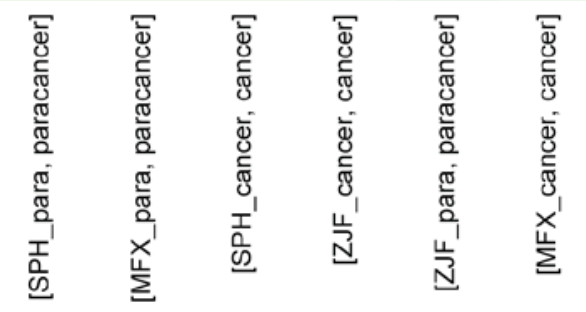

Figure 1. Hierarchical clustering of circRNA expression profiles. The results demonstrate a distinguishable circRNA expression profiling among 6 different samples. The dendrogram indicates the associations between the expression levels of samples. A total of 222,567,556 upregulated circRNAs and $125,439,219$ downregulated circRNAs (fold-change $\geq 2$ ) were identified. circRNA, circular RNA.

Annotation for circRNA/miRNA interactions. The circRNA/miRNA interaction was predicted using the miRNA target prediction software. All differentially expressed circRNAs (fold-change $\geq 2 ; \mathrm{P} \leq 0.05$ ) were annotated in detail

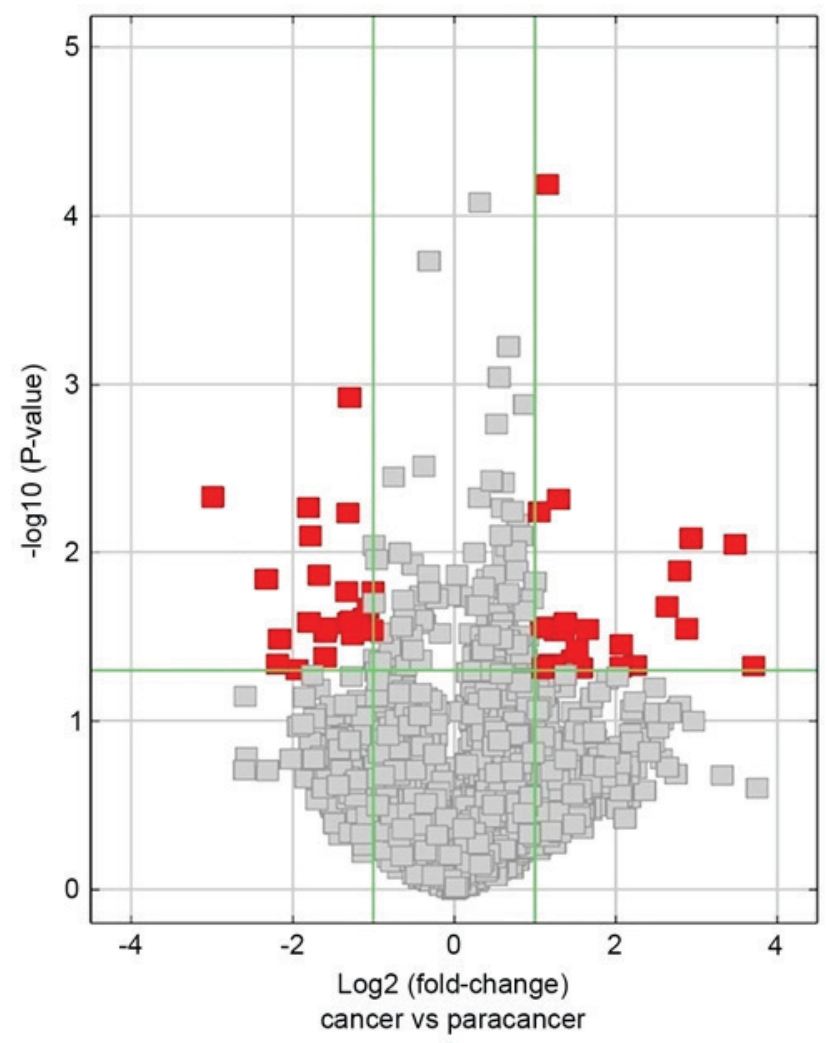

Figure 2. Volcano plot of the expression profiles of dysregulated circRNAs in HCC vs. NT. The vertical green lines correspond to 2 -fold up- and downregulation; and the horizontal line represents, $\mathrm{P}=0.05$. The red points in the plot represent the differentially expressed circRNAs in HCC tissues with statistical significance, compared with NTs (fold-change $\geq 2 ; \mathrm{P} \leq 0.05$ ). A total of 24 upand 23 downregulated circRNAs were identified. circRNAs, circular RNAs; NTs, non-tumorous tissues.

using the circRNA/miRNA interaction information (Tables IV and V). The most upregulated circRNA, hsa_circRNA_104351, adjusts its MREs: hsa-miR-490-5p, hsa-miR-876-5p, hsa-miR-619-3p, hsa-miR-619-3p, hsa-miR-331-3p and hsa-miR-411-3p. Similarly, the most downregulated circRNA, hsa_circRNA_100327, targets the following MREs: Hsa-miR-637, hsa-miR-326, hsa-miR-330-5p, hsa-miR-646 and hsa-miR-24-3p.

\section{Discussion}

Previously, circRNAs have been identified to serve a role in a number of types of disease, including cancer. The majority of circRNAs exhibit distinct tissue/developmental-stage and diseases-specific expression in the process of organismal 


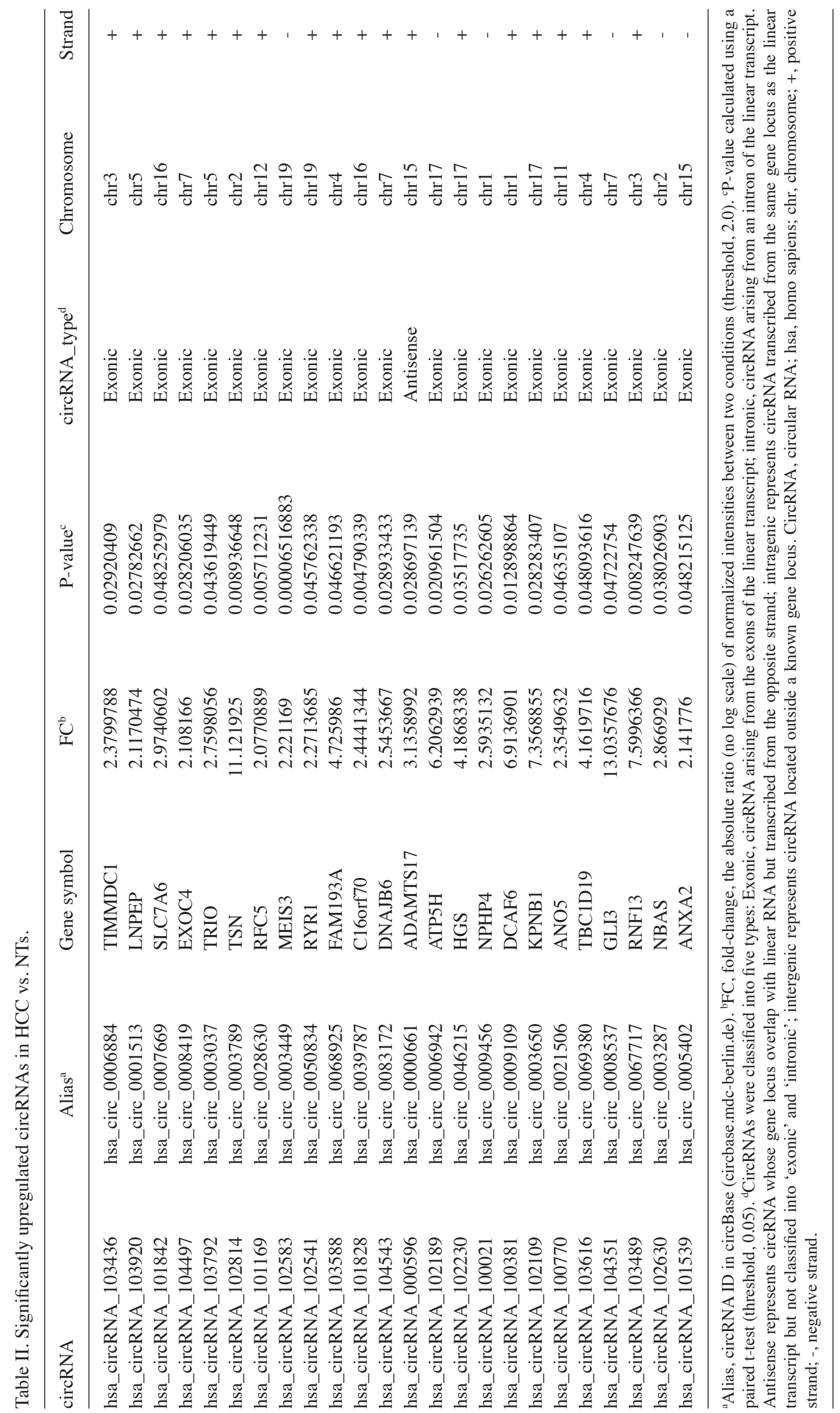




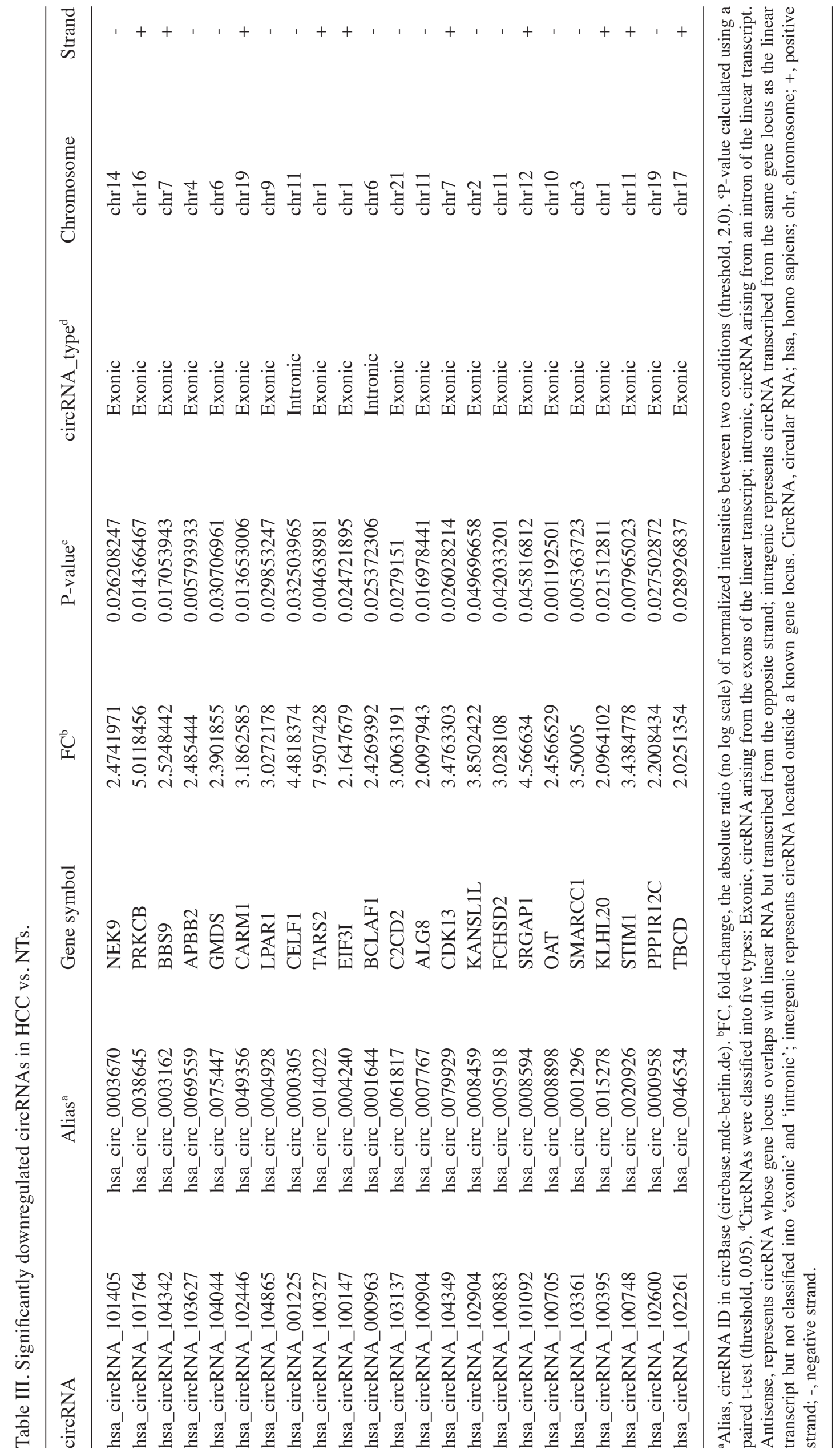




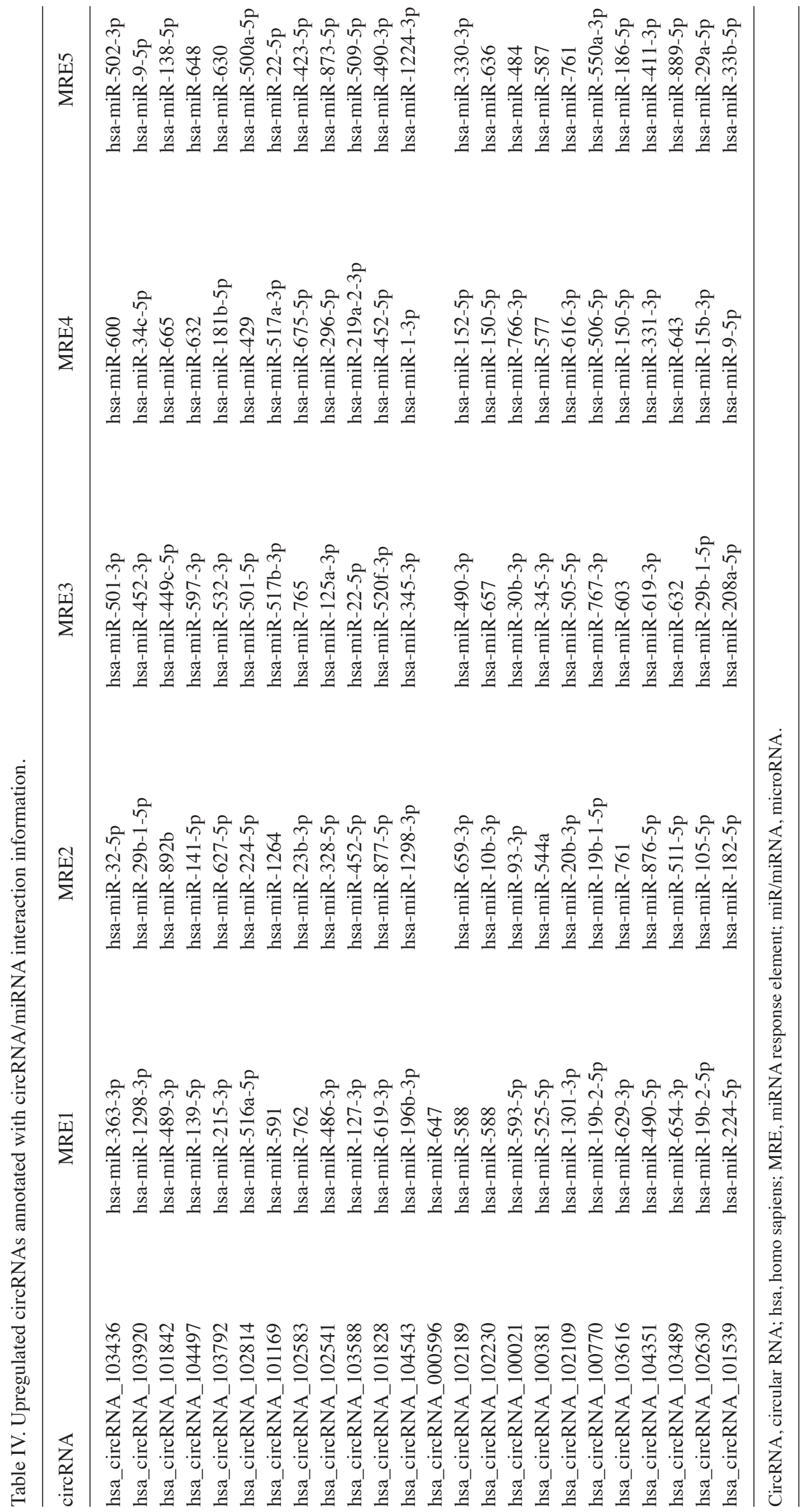




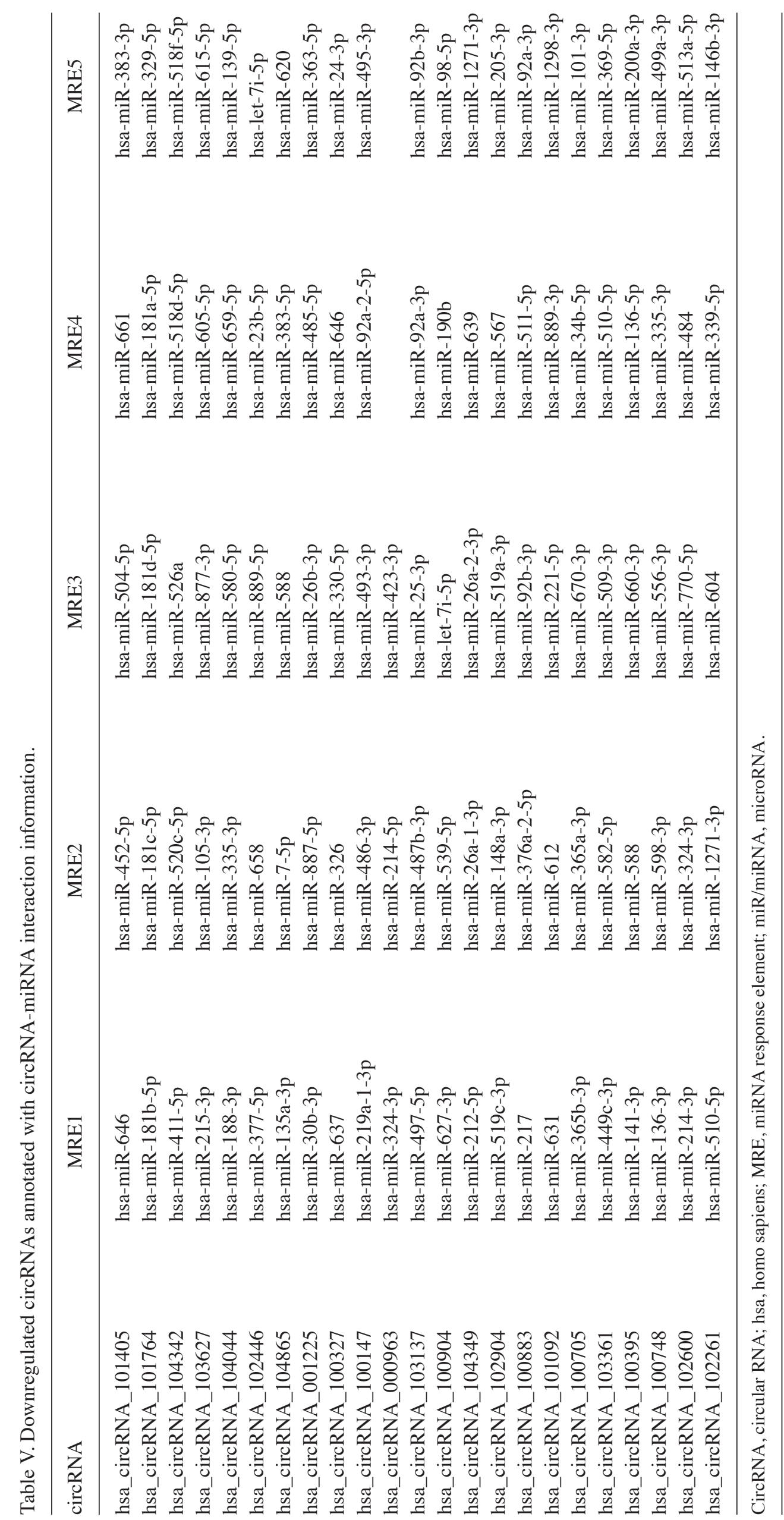


differentiation, development and diseases (6). CircRNAs regulate cancer development via a number of mechanisms, including miRNA sponges, modulating the Wnt signaling pathway and epithelial-mesenchymal transition (23). The abnormal expression levels of circRNAs have been observed in a number of types of cancer (24), including: glioma (25-27), renal cell carcinoma (28), bladder carcinoma (29,30), laryngeal cancer $(31)$, lung cancer $(32,33)$, breast cancer $(34,35)$, esophageal cancer $(36,37)$, gastric cancer $(38-40)$, colorectal cancer (41-46), pancreatic ductal adenocarcinoma (47), cutaneous squamous cell carcinoma (48), basal cell carcinoma (49) and ovarian cancer (50).

Abnormal circRNAs have been identified to be involved in the occurrence and development of HCC (51). In a previous study, the RNA-seq data from 50 paired HCC tissues and NTs were analyzed to identify the function of circRNAs in HCC (51). Protein-coding genes (PCGs) associated with the 2091 circRNAs were identified to be enriched predominantly on liver/cardiovascular-related diseases, and participated in a number of metabolic processes (51). A total of 45 circRNAs and 23 PCGs exhibited significant expression alterations between HCC and normal tissues (51).

Representative circRNA, antisense to cerebellar degeneration-related protein 1 (Cdrlas, also termed ciRS-7), has been identified to act as an oncogene through targeting miR-7 in HCC (52). Cdrlas expression was upregulated and miR-7 expression was downregulated in HCC tissues. Knockdown of Cdrlas downregulated the expression of miR-7, and inhibited the expression of Cyclin E1 (CCNE1) and phosphatidylinositol 3-kinase catalytic subunit delta (PIK3CD), resulting in the suppression of proliferation and invasion of HCC cells through targeting miR-7 (52). Increased Cdrlas expression was significantly associated with hepatic microvascular invasion (MVI), alpha-fetoprotein (AFP) level, younger age and deterioration of HCC. The expression of Cdrlas in HCC tissues with concurrent MVI was inversely associated with miR-7 and positively associated with two miR-7-targeted genes (PIK3CD and p70S6K) (53).

The expression of circZKSCAN1 (zinc finger with KRAB and SCAN domains 1) and ZKSCAN1mRNA was significantly decreased in the HCC samples compared with matched adjacent non-tumorous tissues. The circZKSCAN1 levels varied in patients with tumor numbers, cirrhosis, vascular invasion and the tumor grade. ZKSCAN1mRNA primarily regulated cellular metabolism, whereas circZKSCAN1 mediated a number of cancer-related signaling pathways, suggesting a non-redundant role for ZKSCAN1mRNA and circZKSCAN1 (54).

CircRNAs are abundant, evolutionally conserved and relatively stable in the cytoplasm and therefore may be valuable for cancer diagnosis $(12,14)$. A previous study demonstrated that a total of 174 and 353 circRNAs were upregulated and downregulated in HCC tissues, respectively, according to microarray analysis (55). hsa_circ_0004018 may be involved in cancer-related pathways via interactions with miRNAs (55). hsa_circ_0004018 is downregulated in HCC tissues and HCC lines, and a decreased hsa_circ_0004018 level is associated with serum AFP level, tumor diameters, differentiation, Barcelona Clinic Liver Cancer stage (56) and TNM stage. An additional study identified that hsa_circ_0001649 expression was significantly downregulated in HCC tissues, using the reverse transcription-quantitative polymerase chain reaction (RT-qPCR) (57). hsa_circ_0001649 expression was associated with tumor size and the occurrence of tumor embolus in HCC; therefore, hsa_circ_0001649 may function in the tumorigenesis and metastasis of $\mathrm{HCC}$ and serve as a novel potential biomarker (57). In addition, GO analysis revealed that hsa_circ_0005075 may participate in cell adhesion during HCC development. Upregulated hsa_circ_0005075 exhibited an association with $\mathrm{HCC}$ tumor size and revealed diagnostic potential (58).

The present study compared the circRNA expression profiles between HCC tissue and adjacent NTs using microarray analysis with 5,396 circRNA probes. On the basis of the microarray data, 222,567,556 upregulated circRNAs and 125,439,219 downregulated circRNAs were identified in HCC tissues compared with adjacent NTs. Further analysis identified 24 upregulated and 23 downregulated significantly circRNAs (fold-change $\geq 2 ; \mathrm{P} \leq 0.05$ ) in $\mathrm{HCC}$ tissues compared with NTs. The results of the present study demonstrated that the circRNA expression profiles of HCC tissues differ from that of NTs.

Computer and database analysis annotated the MREs associated with the abnormally expressed circRNAs. The upregulated circRNAs may suppress miRNA expression. On the contrary, downregulated circRNAs may increase miRNA expression. The circRNAs, as a sponge for miRNAs, may be associated with the occurrence and progression of HCC, and may provide a novel approach to identify the underlying molecular basis of HCC. Furthermore, the identified differentially expressed circRNAs may be used as biomarkers for HCC.

In the present study, the abnormal expression levels of three paired HCC tissues were analyzed. Additional studies, with larger cohorts and using RT-qPCR, are required to validate the results from the present study. The data from the present study indicated that abnormal expression of certain circRNAs in HCC tissues and abnormal circRNAs may be novel biomarkers for the diagnosis of HCC.

\section{Acknowledgements}

The present study was supported by the Capital Science and Technology Development Fund (grant no. 2014-1-2181), the Beijing Municipal Administration of Hospitals Clinical Medicine Development of Special Funding (grant no. ZYLX201610), the Liver and AIDS Fund of Beijing YouAn Hospital (grant no. BJYAH-2011-032) and National Sci-Tech Support Plan (grant no. 2015BAI02B00).

\section{References}

1. Zhu RX, Seto WK, Lai CL and Yuen MF: Epidemiology of hepatocellular carcinoma in the Asia-Pacific region. Gut Liver 10: 332-339, 2016.

2. de Martel C, Maucort-Boulch D, Plummer M and Franceschi S: World-wide relative contribution of Hepatitis $B$ and $C$ viruses in hepatocellular carcinoma. Hepatology 62: 1190-1200, 2015.

3. El-Serag HB: Epidemiology of viral hepatitis and hepatocellular carcinoma. Gastroenterology 142: 1264-1273.e1, 2012.

4. Michielsen P and Ho E: Viral hepatitis B and hepatocellular carcinoma. Acta Gastroenterol Belg 74: 4-8, 2011. 
5. Sanger HL, Klotz G, Riesner D, Gross HJ and Kleinschmidt AK : Viroids are single-stranded covalently closed circular RNA molecules existing as highly base-paired rod-like structures. Proc Natl Acad Sci USA 73: 3852-3856, 1976.

6. Memczak S, Jens M, Elefsinioti A, Torti F, Krueger J, Rybak A, Maier L, Mackowiak SD, Gregersen LH, Munschauer M, et al: Circular RNAs are a large class of animal RNAs with regulatory potency. Nature 495: 333-338, 2013.

7. Hsu MT and Coca-Prados M: Electron microscopic evidence for the circular form of RNA in the cytoplasm of eukaryotic cells. Nature 280: 339-340, 1979.

8. Hentze MW and Preiss T: Circular RNAs: Splicing's enigma variations. EMBO J 32: 923-925, 2013.

9. Wilusz JE and Sharp PA: Molecular biology. A circuitous route to noncoding RNA. Science 340: 440-441, 2013.

10. Wu Q, Wang Y, Cao M, Pantaleo V, Burgyan J, Li WX and Ding SW: Homology-independent discovery of replicating pathogenic circular RNAs by deep sequencing and a new computational algorithm. Proc Natl Acad Sci USA 109: 3938-3943, 2012.

11. Salzman J, Gawad C, Wang PL, Lacayo N and Brown PO: Circular RNAs are the predominant transcript isoform from hundreds of human genes in diverse cell types. PLoS One 7 : e30733, 2012.

12. Jeck WR, Sorrentino JA, Wang K, Slevin MK, Burd CE, Liu J, Marzluff WF and Sharpless NE: Circular RNAs are abundant, conserved, and associated with ALU repeats. RNA 19: 141-157, 2013.

13. Zhang Y, Zhang XO, Chen T, Xiang JF, Yin QF, Xing YH, Zhu S, Yang L and Chen LL: Circular intronic long noncoding RNAs. Mol Cell 51: 792-806, 2013.

14. Hansen TB, Jensen TI, Clausen BH, Bramsen JB, Finsen B Damgaard CK and Kjems J: Natural RNA circles function as efficient microRNA sponges. Nature 495: 384-388, 2013.

15. Guo JU, Agarwal V, Guo H and Bartel DP: Expanded identification and characterization of mammalian circular RNAs. Genome Biol 15: 409, 2014.

16. Wilusz JE and Sharp PA: Molecular biology. A circuitous route to noncoding RNA. Science 340: 440-441, 2013.

17. Ghosal S, Das S, Sen R, Basak P and Chakrabarti J: Circ2Traits: A comprehensive database for circular RNA potentially associated with disease and traits. Front Genet 4: 283, 2013.

18. Burd CE, Jeck WR, Liu Y, Sanoff HK, Wang Z and Sharpless NE: Expression of linear and novel circular forms of an INK4/ARF-associated non-coding RNA correlates with atherosclerosis risk. PLoS Genet 6: e1001233, 2010.

19. Chen YT, Rettig WJ, Yenamandra AK, Kozak CA, Chaganti RS, Posner JB and Old LJ: Cerebellar degeneration-related antigen: A highly conserved neuroectodermal marker mapped to chromosomes X in human and mouse. Proc Natl Acad Sci USA 87: 3077-3081, 1990 .

20. Hansen TB, Kjems J and Damgaard CK: Circular RNA and miR-7 in cancer. Cancer Res 73: 5609-5612, 2013.

21. Li J, Yang J, Zhou P, Le Y, Zhou C, Wang S, Xu D, Lin HK and Gong Z: Circular RNAs in cancer: Novel insights into origins, properties, functions and implications. Am J Cancer Res 5: 472-480, 2015

22. Okuda K, Ohtsuki T, Obata H, Tomimatsu M, Okazaki N, Hasegawa H, Nakajima $Y$ and Ohnishi K: Natural history of hepatocellular carcinoma and prognosis in relation to treatment. Study of 850 patients. Cancer 56 918-928, 1985

23. He J, Xie Q, Xu H, Li J and Li Y: Circular RNAs and cancer. Cancer Lett 396: 138-144, 2017.

24. Rong D, Tang W, Li Z, Zhou J, Shi J, Wang H and Cao H: Novel insights into circular RNAs in clinical application of carcinomas. Onco Targets Ther 10: 2183-2188, 2017.

25. Song X, Zhang N, Han P, Moon BS, Lai RK, Wang K and Lu W: Circular RNA profile in gliomas revealed by identification tool UROBORUS. Nucleic Acids Res 44: e87, 2016.

26. Yang P, Qiu Z, Jiang Y, Dong L, Yang W, Gu C, Li G and Zhu Y: Silencing of cZNF292 circular RNA suppresses human glioma tube formation via the $\mathrm{Wnt} / \beta$-catenin signaling pathway. Oncotarget 7: 63449-63455, 2016.

27. Barbagallo D, Condorelli A, Ragusa M, Salito L, Sammito M, BanelliB,CaltabianoR,Barbagallo G,Zappalà A,BattagliaR, etal Dysregulated miR-671-5p/CDR1-AS/CDR1/VSNL1 axis is involved in glioblastoma multiforme. Oncotarget 7: 4746-4759, 2016.
28. Wang K, Sun Y, Tao W, Fei X and Chang C: Androgen receptor (AR) promotes clear cell renal cell carcinoma (ccRCC) migration and invasion via altering the circHIAT1/miR-195-5p/29a-3p/29c-3p/CDC42 signals. Cancer Lett 394: 1-12, 2017.

29. Zhong Z, Lv M and Chen J: Screening differential circular RNA expression profiles reveals the regulatory role of circTCF25-miR-103a-3p/miR-107-CDK6 pathway in bladder carcinoma. Sci Rep 6: 30919, 2016.

30. Huang M, Zhong Z, Lv M, Shu J, Tian Q and Chen J: Comprehensive analysis of differentially expressed profiles of lncRNAs and circRNAs with associated co-expression and competing endogenous RNAs networks in bladder carcinoma. Oncotarget 7: 47186-47200, 2016.

31. Xuan LJ, Qu LM, Zhou H, Wang P, Yu H, Wu T, Wang X, Li Q, Tian L, Liu M and Sun Y. Circular RNA: A novel biomarker for progressive laryngeal cancer. Am J Transl Res 8: 932-939, 2016.

32. Yao JT, Zhao SH, Liu QP, Lv MQ, Zhou DX, Liao ZJ and Nan KJ: Over-expression of CircRNA-100876 in non-small cell lung cancer and its prognostic value. Pathol Res Pract 213: 453-456, 2017.

33. Wan L, Zhang L, Fan K, Cheng ZX, Sun QC and Wang JJ: Circular RNA-ITCH suppresses lung cancer proliferation via inhibiting the Wnt/ $\beta$;-catenin pathway. Biomed Res Int 2016 $1579490,2016$.

34. Lü L, Sun J, Shi P, Kong W, Xu K, He B, Zhang S and Wang J: Identification of circular RNAs as a promising new class of diagnostic biomarkers for human breast cancer. Oncotarget 8: 44096-44107, 2017.

35. Nair AA, Niu N, Tang X, Thompson KJ, Wang L, Kocher JP, Subramanian S and Kalari KR: Circular RNAs and their associations with breast cancer subtypes. Oncotarget 7: 80967-80979, 2016.

36. Su H, Lin F, Deng X, Shen L, Fang Y, Fei Z, Zhao L, Zhang X, Pan H, Xie D, et al: Profiling and bioinformatics analyses reveal differential circular RNA expression in radioresistant esophageal cancer cells. J Transl Med 14: 225, 2016.

37. Li F, Zhang L, Li W, Deng J, Zheng J, An M, Lu J and Zhou Y: Circular RNA ITCH has inhibitory effect on ESCC by suppressing the Wnt/ $\beta$-catenin pathway. Oncotarget 6 : 6001-6013, 2015 .

38. Li P, Chen S, Chen H, Mo X, Li T, Shao Y, Xiao B and Guo J: Using circular RNA as a novel type of biomarker in the screening of gastric cancer. Clin Chim Acta 444: 132-136, 2015.

39. Li P, Chen H, Chen S, Mo X, Li T, Xiao B, Yu R and Guo J: Circular RNA 0000096 affects cell growth and migration in gastric cancer. Br J Cancer 116: 626-633, 2017

40. Chen S, Li T, Zhao Q, Xiao B and Guo J: Using circular RNA hsa_circ_0000190 as a new biomarker in the diagnosis of gastric cancer. Clin Chim Acta 466: 167-171, 2017.

41. Tang W, Ji M, He G, Yang L, Niu Z, Jian M, Wei Y, Ren L and $\mathrm{Xu}$ J: Silencing CDR las inhibits colorectal cancer progression through regulating microRNA-7. Onco Targets Ther 10 2045-2056, 2017

42. Huang G, Zhu H, Shi Y, Wu W, Cai H and Chen X: cir-ITCH plays an inhibitory role in colorectal cancer by regulating the Wnt/ $\beta$-catenin pathway. PLoS One 10: e0131225, 2015.

43. Wang X, Zhang Y, Huang L, Zhang J, Pan F, Li B, Yan Y, Jia B, Liu H, Li S and Zheng W: Decreased expression of hsa_ circ 001988 in colorectal cancer and its clinical significances. Int J Clin Exp Pathol 8: 16020-16025, 2016.

44. Xie H, Ren X, Xin S, Lan X, Lu G, Lin Y, Yang S, Zeng Z, Liao W, Ding Y and Liang L: Emerging roles of circRNA 001569 targeting miR-145 in the proliferation and invasion of colorectal cancer. Oncotarget 7: 26680-26691, 2016.

45. Zhu M, Xu Y, Chen Y and Yan F: Circular BANP, an upregulated circular RNA that modulates cell proliferation in colorectal cancer. Biomed Pharmacother 88: 138-144, 2017.

46. Dou Y, Cha DJ, Franklin JL, Higginbotham JN, Jeppesen DK, Weaver AM, Prasad N, Levy S, Coffey RJ, Patton JG and Zhang B: Circular RNAs are down-regulated in KRAS mutant colon cancer cells and can be transferred to exosomes. Sci Rep 6: 37982, 2016.

47. Qu S, Song W, Yang X, Wang J, Zhang R, Zhang Z, Zhang H and $\mathrm{Li} \mathrm{H}$ : Microarray expression profile of circular RNAs in human pancreatic ductal adenocarcinoma. Genom Data 5: 385-387, 2015.

48. Sand M, Bechara FG, Gambichler T, Sand D, Bromba M, Hahn SA, Stockfleth E and Hessam S: Circular RNA expression in cutaneous squamous cell carcinoma. J Dermatol Sci 83: 210-218, 2016. 
49. Sand M, Bechara FG, Sand D, Gambichler T, Hahn SA, Bromba M, Stockfleth E and Hessam S: Circular RNA expression in basal cell carcinoma. Epigenomics 8: 619-632, 2016.

50. Ahmed I, Karedath T, Andrews SS, Al-Azwani IK Mohamoud YA, Querleu D, Rafii A and Malek JA: Altered expression pattern of circular RNAs in primary and metastatic sites of epithelial ovarian carcinoma. Oncotarget 7: 36366-36381, 2016.

51. Li Y, Dong Y, Huang Z, Kuang Q, Wu Y, Li Y and Li M: Computational identifying and characterizing circular RNAs and their associated genes in hepatocellular carcinoma. PLoS One 12: e0174436, 2017.

52. Yu L, Gong X, Sun L, Zhou Q, Lu B and Zhu L: The circular RNA Cdrlas act as an oncogene in hepatocellular carcinoma through targeting miR-7 expression. PLoS One 11: e0158347, 2016.

53. Xu L, Zhang M, Zheng X, Yi P, Lan C and Xu M: The circular RNA ciRS-7 (Cdrlas) acts as a risk factor of hepatic microvascular invasion in hepatocellular carcinoma. J Cancer Res Clin Oncol 143: 17-27, 2017.
54. Yao Z, Luo J, Hu K, Lin J, Huang H, Wang Q, Zhang P, Xiong Z, He C, Huang Z, et al: ZKSCAN1 gene and its related circular RNA (circZKSCAN1) both inhibit hepatocellular carcinoma cell growth, migration, and invasion but through different signaling pathways. Mol Oncol 11: 422-437, 2017.

55. Fu L, Yao T, Chen Q, Mo X, Hu Y and Guo J: Screening differential circular RNA expression profiles reveals hsa_circ_0004018 is associated with hepatocellular carcinoma. Oncotarget 8: 58405-58416, 2017.

56. Bruix J and Sherman M; American Association for the Study of Liver Diseases: Management of hepatocellular carcinoma: An update. Hepatology 53: 1020-1022, 2011.

57. Qin M, Liu G, Huo X, Tao X, Sun X, Ge Z, Yang J, Fan J, Liu L and Qin W: Hsa_circ_0001649: A circular RNA and potential novel biomarker for hepatocellular carcinoma. Cancer Biomark 16: 161-169, 2016

58. Shang X, Li G, Liu H, Li T, Liu J, Zhao Q and Wang C: Comprehensive circular RNA profiling reveals that hsa circ_0005075, a new circular RNA biomarker, is involved in hepatocellular carcinoma development. Medicine 95: e3811, 2016. 